\title{
Attenuation of indirect markers of eccentric exercise-induced muscle damage by curcumin
}

\author{
Yoko Tanabe $^{1} \cdot$ Seiji Maeda $^{2}$ - Nobuhiko Akazawa ${ }^{2}$ Asako Zempo-Miyaki ${ }^{3}$. \\ Youngju Choi $^{2} \cdot$ Song-Gyu $\mathrm{Ra}^{2} \cdot$ Atsushi Imaizumi $^{4} \cdot$ Yoshihiko Otsuka $^{4}$. \\ Kazunori Nosaka ${ }^{5}$
}

Received: 4 June 2014 / Accepted: 6 April 2015 / Published online: 29 April 2015

(C) The Author(s) 2015. This article is published with open access at Springerlink.com

\begin{abstract}
Purpose Polyphenolic curcumin is known to have potent anti-inflammatory effects; thus the present study investigated the hypothesis that curcumin ingestion would attenuate muscle damage after eccentric exercise.

Methods Fourteen untrained young men ( $24 \pm 1$ years) performed 50 maximal isokinetic $(120 \%$ s) eccentric contractions of the elbow flexors of one arm on an isokinetic dynamometer and the same exercise with the other arm 4 weeks later. They took $150 \mathrm{mg}$ of curcumin (theracurmin) or placebo (starch) orally before and $12 \mathrm{~h}$ after each eccentric exercise bout in a randomised, crossover design. Maximal voluntary contraction (MVC) torque of the elbow flexors, range of motion of the elbow joint, upper-arm circumference, muscle soreness, serum creatine kinase (CK) activity, and plasma interleukin-6 (IL-6) and tumor necrosis factor- $\alpha$ (TNF- $\alpha)$ concentration were measured before, immediately after, and 24, 48, 72 and $96 \mathrm{~h}$ after each eccentric exercise. Changes in these variables over time
\end{abstract}

Communicated by Fabio Fischetti

Seiji Maeda

maeda@taiiku.tsukuba.ac.jp

1 Graduate School of Comprehensive Human Science, University of Tsukuba, Tsukuba, Ibaraki, Japan

2 Faculty of Health and Sport Sciences, University of Tsukuba, 1-1-1 Tennodai, Tsukuba, Ibaraki 305-8574, Japan

3 Faculty of Medicine, University of Tsukuba, Tsukuba, Ibaraki, Japan

4 Theravalues Corporation, Tokyo, Japan

5 Centre for Exercise and Sports Science Research, Edith Cowan University, Joondalup, WA, Australia were compared between curcumin and placebo conditions by two-way repeated measures ANOVA.

Results MVC torque decreased smaller and recovered faster (e.g., 4 days post-exercise: $-31 \pm 13 \%$ vs. $-15 \pm 15 \%$ ), and peak serum CK activity was smaller (peak: $7684 \pm 8959$ IU/L vs. $3398 \pm 3562$ IU/L) for curcumin than placebo condition $(P<0.05)$. However, no significant differences between conditions were evident for other variables, and no significant changes in IL- 6 and TNF- $\alpha$ were evident after exercise.

Conclusion It is concluded that theracurmin ingestion attenuates some aspects of muscle damage such as MVC loss and CK activity increase.

Keywords Polyphenolic $\cdot$ Elbow flexors $\cdot$ Muscle strength $\cdot$ Muscle soreness $\cdot$ Supplement $\cdot$ Creatine kinase

$\begin{array}{ll}\text { Abbreviations } \\ \text { CK } & \text { Creatine kinase } \\ \text { DOMS } & \text { Delayed onset muscle soreness } \\ \text { IL-6 } & \text { Interleukin 6 } \\ \text { MVC } & \text { Maximal voluntary contraction } \\ \text { ROM } & \text { Range of motion } \\ \text { TNF- } \alpha & \text { Tumor necrosis factor- } \alpha \\ \text { VAS } & \text { Visual analog scale }\end{array}$

\section{Introduction}

Unaccustomed exercise, especially that consists of highintensity and/or large number of eccentric (lengthening) contractions, induces muscle damage (Clarkson et al. 1992). Eccentric exercise-induced muscle damage is indicated by prolonged decreases in muscle strength and range of motion (ROM), swelling, delayed onset muscle soreness 
(DOMS), and increased muscle proteins in the blood such as creatine kinase (CK) activity (Warren et al. 1999). Muscle damage initiates inflammatory responses, resulting in secondary muscle damage (Tiidus 1998). The impairment of muscle function caused by damage and subsequent inflammatory responses could reduce the ability to perform daily activities and athletic performance. Thus, it is important to prevent or minimise muscle damage after exercise by attenuating inflammatory responses. One of many measures for this purpose could be an oral intake of anti-inflammatory substances (Connolly et al. 2003).

Several studies have shown that some anti-inflammatory substance supplementations are beneficial for attenuating eccentric exercise-induced muscle damage (Connolly et al. 2006; Howatson et al. 2010; Trombold et al. 2010), but other did not find such effect (O'Fallon et al. 2012). These inconsistent results may, in part, be due to the differences in substances, exercise model and experimental design among studies. It has been reported that tart cherry juice consumption enhanced recovery of muscle strength and attenuated interleukin-6 (IL-6) and C-reactive protein responses after marathon running (Howatson et al. 2010) and prolonged upper arm exercise (Connolly et al. 2006). Other study showed that taking polyphenol ellagitannins from pomegranate extract before and after high-intensity elbow flexor eccentric exercise attenuated muscle strength loss by $10 \%$ (Trombold et al. 2010). In contrast, O'Fallon et al. (2012) reported that ingestion of a polyphenol flavonoid from quercetin before and after exercise did not affect changes in maximal voluntary contraction (MVC) strength, ROM, serum CK activity, IL-6 and tumor necrosis factor- $\alpha$ (TNF$\alpha)$ and DOMS after elbow flexor eccentric contractions.

Curcumin, extracted from turmeric, is a natural polyphenolic substance. It has been demonstrated that curcumin has anti-inflammatory effect by downregulating the nuclear factor-kappa B (NF-kB), thereby suppressing the expression of IL-6 and TNF- $\alpha$ (Cho et al. 2007; Aggarwal et al. 2006). The anti-inflammatory effects of curcumin have been used in the treatment of rheumatoid arthritis, inflammatory bowel disease, multiple sclerosis and diabetes (Sethi et al. 2009). Usharani et al. (2008) demonstrated that $600 \mathrm{mg}$ of daily curcumin intake for 8 weeks reduced approximately $60 \%$ of plasma IL- 6 and TNF- $\alpha$ concentrations in diabetes mellitus patients. Polyphenol supplementation of curcumin has been reported to blunt IL- 6 and $\mathrm{TNF}-\alpha$ increases in mice muscle after downhill running (Davis et al. 2007). Kawanishi et al. (2013) have recently reported that oral curcumin ingestion $(3 \mathrm{mg}$ ) suppresses oxidative stress, reducing hydrogen peroxide in skeletal muscle following downhill running-induced muscle damage in mice. Thus, it is possible that curcumin supplementation is effective for attenuating eccentric exercise-induced muscle damage in humans. However, to the best of our knowledge, no previous human study has examined the effects of curcumin ingestion on markers of muscle damage after eccentric exercise.

The purpose of this study, therefore, was to examine the acute effect of curcumin intake before and $12 \mathrm{~h}$ postexercise on changes in indirect markers of muscle damage and inflammation after eccentric elbow flexors exercise. It was hypothesised that curcumin ingestion would attenuate the changes in indirect muscle damage markers and inflammatory markers in the blood after eccentric exercise when compared with placebo condition.

\section{Methods}

\section{Subjects}

The present study recruited 14 healthy, untrained young men who had not been involved in any regular resistance training for at least 1 year before this study. Their average \pm SD age, height and body mass were $23.5 \pm 2.3$ years, $172.1 \pm 7.5 \mathrm{~cm}$ and $65.2 \pm 11.3 \mathrm{~kg}$, respectively. The sample size was estimated on the assumption that the curcumin ingestion could attenuate the decrease in muscle strength in recovery days by at least $25 \%$, if it was effective. On the basis of the power of 0.80 and alpha level of 0.05 , a sample size of 10 was found to be necessary based on the data of a previous study (Evans et al. 2002) in which a similar eccentric exercise to that of the present study was performed. Subjects maintained their normal food intake and lifestyle habits, but abstained from strenuous physical activities, and did not take anti-inflammatory drugs during the study period. All subjects gave their written informed consent to participate in the study, and all procedures were reviewed and approved by the ethical committee of the University of Tsukuba.

\section{Experimental design}

This study used a crossover design in which one arm was used for curcumin condition, and the other arm was used for placebo condition in a randomised, single-blinded fashion. This design requires a smaller number of participants, and it has been reported that a within-subject (contralateral arm) design is an alternative to a between-subject design if the order of exercise is counterbalanced by arm dominance (Newton et al. 2013).

Each arm performed a bout of eccentric exercise of the elbow flexors separated by 4 weeks, and the use of dominant-nondominant arms and the order of the conditions were counterbalanced among the subjects. Dependent variables consisted of MVC torque of the elbow flexors, ROM of the elbow joint, upper-arm circumference, muscle 
soreness, serum CK activity, and plasma IL- 6 and TNF- $\alpha$ concentrations. Changes in these variables before, immediately after and 24, 48, 72 and $96 \mathrm{~h}$ after the exercise were compared between the curcumin and placebo conditions.

\section{Curcumin and placebo}

We developed theracurmin (Theravalues Corporation, Japan) using a microparticulation and surface processing technique, and theracurmin has been shown to result in much higher plasma concentration and bioavailability after intake when compared with conventional curcumin powder (Sasaki et al. 2011). As a placebo, starch was used, which was contained in an identical capsule as that of the curcumin, so the subjects could not distinguish between them. Six capsules of $25 \mathrm{mg}$ of curcumin or placebo were administered 1 hour before $(150 \mathrm{mg})$ and $12 \mathrm{~h}$ after exercise $(150 \mathrm{mg})$. It has been reported that postexercise inflammation starts to present around 4-12 $\mathrm{h}$ after eccentric exercise, and further develops in $24-48 \mathrm{~h}$ after exercise (Paulsen et al. 2012; Davis et al. 2007). Considering the time taken for the curcumin to get into the blood stream, and its remaining time in the blood, it was assumed that the ingestion at $1 \mathrm{~h}$ before exercise could attenuate the initial inflammation, and that at $12 \mathrm{~h}$ after exercise could attenuate the development of inflammation before $24 \mathrm{~h}$ post-exercise. The amount $(150 \mathrm{mg})$ has been shown to be safe for one time dose (Kanai et al. 2012). However, it had not been known before this study how much of curcumin would be effective to prevent muscle damage in humans, so we assumed that a significant difference between the curcumin and placebo conditions would be shown by the dose (150 $\mathrm{mg}$ before and $12 \mathrm{~h}$ after exercise, $300 \mathrm{mg} /$ day), if any positive effect of curcumin ingestion on muscle damage existed.

\section{Eccentric exercise}

All subjects performed two bouts of eccentric exercise of the elbow flexors on a BIODEX dynamometer (BIODEX System 3, USA), using one arm for each bout separated by 4 weeks. Each subject was seated on the dynamometer chair with the arm being supported by padded armrest secured at $45^{\circ}$ shoulder flexion and the waist and chest being stabilised with straps. The exercise consisted of 50 maximal eccentric contractions of the elbow flexors at an angular velocity $120 \%$, and the range of motion was from $130^{\circ}$ and to $10^{\circ}$ elbow flexion, where a fully extended elbow joint angle was considered to be $0^{\circ}$, thus each contraction time was $1 \mathrm{~s}$ (Evans et al. 2002). The exercise limb was passively returned to the initial position at $10 \%$, creating a 12-s rest between contractions. The subjects were instructed to contract the elbow flexors maximally to resist the elbow extending action of the dynamometer for the whole range of motion. Total work and peak torque were calculated using the BIODEX System software.

\section{Muscle damage markers}

Several indirect markers of muscle damage, which were often used in previous studies (Nosaka et al. 2002a, b), were measured before, immediately after, and 24, 48, 72 and $96 \mathrm{~h}$ after each exercise bout. The details of the markers are shown below. The test-retest reliability of each measure was determined using two baseline measurements performed before exercise by an interclass correlation coefficient $(R)$ and coefficient of variation $(\mathrm{CV})$. The $R$ and $\mathrm{CV}$ (shown in parentheses) values for MVC torque, ROM and upper-arm circumference were $0.99(2.9 \%), 0.97(1.1 \%)$ and $1.00(0.2 \%)$, respectively. The muscle soreness was not assessed for the reliability, because all participants recorded DOMS scores as zero for palpation during the familiarisation sessions. The intra-assay $\mathrm{CV}$ was $5.3 \%$ for CK activity, $3.2 \%$ for IL- 6 and $10.0 \%$ for TNF- $\alpha$ in a pilot study using five men.

\section{MVC torque}

MVC torque of the exercised elbow flexors was measured using the isokinetic dynamometer in the same positioning as that of the eccentric exercise, and the elbow joint angle was set at $90^{\circ}$. Three 5-s maximal isometric contractions were performed with a 30-s rest between trials, and the highest value of the three trials was used for further analysis (Nosaka et al. 2002a).

\section{ROM}

According to the protocol of the previous study (Nosaka et al. 2006), each subject was asked to actively extend the elbow joint maximally (extended elbow joint angle) and to touch shoulder of the same side with the hand (flexed elbow joint angle). The ROM was determined as the difference between the two elbow joint angles. Each angle was measured by a goniometer three times, and the mean of the three measures was used for the calculation of ROM.

\section{Upper-arm circumference}

To assess muscle swelling, upper-arm circumference was assessed at the mid-belly of biceps brachii muscle $(60 \%$ distal to the length between the acromial process and epicondylus lateralis humeri) using a standard tape measure (Evans et al. 2002). The measurements were taken three times, and mean value of the three measurements was used for further analysis. 


\section{Muscle soreness}

Muscle soreness upon palpation of the upper-arm and passively extending the elbow joint was quantified by a visual analog scale (VAS) that had a 100-mm line with "no pain" on one end and "extremely sore" on the other end (Chen and Nosaka 2006). The subjects were instructed to sit with arm relaxed, and the investigator palpated over the biceps brachii, and extended the elbow joint maximally. The same investigator performed all tests and the pressure of palpation was kept as constant as possible between subjects and between days.

\section{Blood sampling and analyses}

Blood was taken from the antecubital vein by a standard venipuncture technique. To obtain serum for CK activity analysis, $9 \mathrm{ml}$ blood sample was collected using commercially produced vacuum-sealed serum collection tube (SEKISUI Medical Company Limited, Japan). To obtain the plasma for curcumin, IL-6, and TNF- $\alpha$ analyses, $5 \mathrm{ml}$ blood sample was collected using a tube containing ethlenediaminetetraacetic acid (Terumo corporation, Japan). These were centrifuged at $3000 \mathrm{rpm}$ for $15 \mathrm{~min}$ at $4{ }^{\circ} \mathrm{C}$, and the serum and plasma samples were stored at $-80{ }^{\circ} \mathrm{C}$ until analysis. Plasma curcumin concentration was measured by a HPLC-MS/MS system consisting of a Prominence micro-C system (Shimadzu, Kyoto, Japan) and an API 3200 tandem mass spectrometer (Applied Biosystems CA, USA). Serum CK activity was measured using a test kit (L-type CK, Wako Pure Chemical Industries, Japan) by a commercial laboratory. The normal reference range for male adults with this method is $48-259 \mathrm{IU} / \mathrm{L}$. The concentrations of plasma IL- 6 and TNF- $\alpha$ were determined using a commercial enzyme-linked immunosorbent assay kit (Quantikine Human IL-6, TNF- $\alpha$ R \& D system Inc. Minneapolis, USA).

\section{Statistical analyses}

Changes in the dependent variables over time were compared between the curcumin and placebo conditions by a two-way repeated-measures ANOVA. When the ANOVA showed a significant interaction effect, a Tukey's post hoc test was used to locate differences between conditions. A Tukey's post hoc test was also used to compare the preexercise and post-exercise values for each bout separately, when a significant time effect was found by the ANOVA. Inter-group comparison for the total work and mean eccentric peak torque during the exercise were made by a paired $t$ test. Since a Kolmogorov-Smirov test showed that serum CK activity data was not normally distributed, the peak

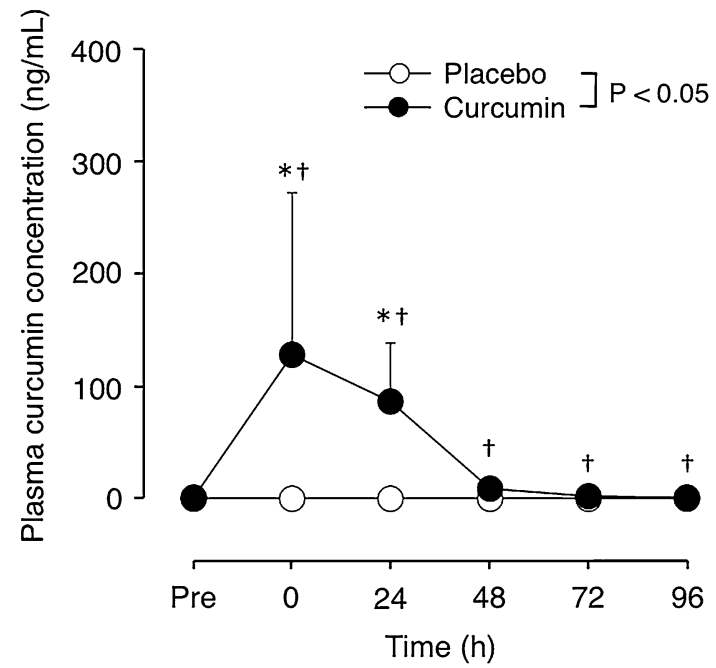

Fig. 1 Changes (mean \pm SD) in plasma curcumin concentration from the baseline (Pre), immediately (0) and 24-96 h after eccentric exercise of the elbow flexors for the curcumin and placebo supplementation conditions. ${ }^{*} P<0.05$ vs. Pre; ${ }^{\dagger} P<0.05$ vs. placebo; $P<0.05$ : a significant interaction effect

serum CK activity was compared between the condition using a non-parametric Wilcoxon's rank test. The level of statistical significance was set at $P<0.05$. All data are shown as mean \pm standard deviation (SD).

\section{Results}

\section{Plasma curcumin concentration}

There was no difference in plasma curcumin concentration at baseline between curcumin and placebo conditions, but a significant interaction effect was evident for the changes after exercise (Fig. 1). Plasma curcumin concentration increased from baseline $(0.04 \pm 0.07 \mathrm{ng} / \mathrm{mL})$ to $127.7 \pm 144.6,85.7 \pm 51.6,8.6 \pm 5.5,2.2 \pm 1.4$ and $0.9 \pm 0.6 \mathrm{ng} / \mathrm{mL}$ at $0,24,48,72$ and $96 \mathrm{~h}$ after exercise, respectively, for the curcumin condition. However, in the placebo condition, no significant changes in plasma curcumin concentration were observed from the baseline $(0.01 \pm 0.05 \mathrm{ng} / \mathrm{mL})$ at any time points after exercise $(0.02 \pm 0.05,0.02 \pm 0.04,0.03 \pm 0.08,0.03 \pm 0.04$ and $0.01 \pm 0.02 \mathrm{ng} / \mathrm{mL}$ at $0,24,48,72$ and $96 \mathrm{~h}$ post-exercise, respectively).

\section{Exercise}

The total work $(2815 \pm 788 \mathrm{~J}$ vs. $3011 \pm 756 \mathrm{~J}, P=0.178)$ and mean peak torque $(40 \pm 11 \mathrm{Nm}$ vs. $41 \pm 9 \mathrm{Nm}$, $P=0.597)$ during eccentric exercise did not differ between curcumin and placebo conditions. 


\section{MVC torque}

The MVC torque before exercise was not significantly different between curcumin $(52.5 \pm 10.3 \mathrm{Nm})$ and placebo $(55.1 \pm 8.8 \mathrm{Nm})$ conditions. As shown in Fig. 2, a significant interaction effect was evident for the changes in MVC torque. The change in MVC from baseline was significantly smaller for the curcumin condition $(33.0 \pm 8.0 \%)$ compared with placebo condition $(40.0 \pm 9.1 \%)$ at immediately after exercise and also at $48-96 \mathrm{~h}$ after exercise by $13-16 \%$.

\section{ROM}

At the baseline, ROM was similar between curcumin $\left(134^{\circ} \pm 4^{\circ}\right)$ and placebo $\left(133^{\circ} \pm 5^{\circ}\right)$ conditions. After eccentric exercise, ROM significantly decreased at all measurement time points from the baseline, but no significant interaction effect was evident for the changes between conditions (Fig. 3).

\section{Upper-arm circumference}

There was no significant difference in the baseline upperarm circumference between curcumin $(25.7 \pm 2.5 \mathrm{~cm})$ and placebo $(25.9 \pm 2.8 \mathrm{~cm})$ conditions. Upper-arm circumference significantly increased after exercise by $3 \%$ at $96 \mathrm{~h}$ post-exercise for both conditions, but no significant differences between conditions were found (Fig. 4).

\section{Muscle soreness}

Significant increases in VAS were observed between 24 and $96 \mathrm{~h}$ after eccentric exercise for both conditions, and the peak VAS upon palpation was $5.5 \pm 2.1 \mathrm{~cm}$ for curcumin and $6.0 \pm 2.2 \mathrm{~cm}$ for placebo condition, but no significant interaction effect was evident (Fig. 5). This was also the case for the VAS with elbow joint extension, and the values were similar to those shown by the palpation.

\section{Serum CK activity}

Serum CK activity was within normal reference range prior to exercise without any significant difference between the curcumin $(118 \pm 49 \mathrm{IU} / \mathrm{L})$ and control $(115 \pm 47 \mathrm{IU} / \mathrm{L})$ conditions. Although the changes in serum $\mathrm{CK}$ activity after eccentric exercise were not statistically different between conditions (Fig. 6, $P=0.052$ ), peak CK activity was significantly smaller for curcumin condition $(3398 \pm 3562$ IU/L) than placebo condition $(7684 \pm 8959 \mathrm{IU} / \mathrm{L})$.

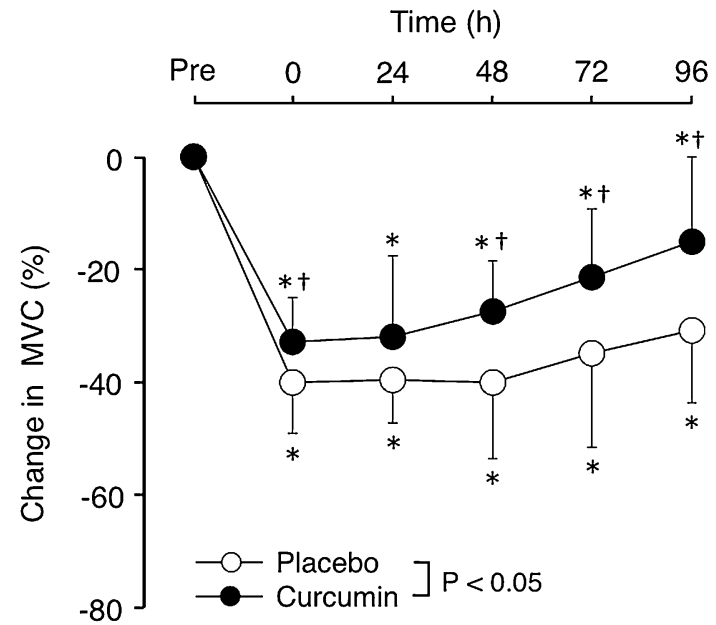

Fig. 2 Normalised changes (mean $\pm \mathrm{SD}$ ) in maximal voluntary isometric contraction (MVC) torque from the baseline (Pre), at immediately (0) and 24-96 h after eccentric exercise of the elbow flexors for the curcumin and placebo supplementation conditions. $* P<0.05$ vs. Pre; ${ }^{\dagger} P<0.05$ vs. placebo; $P<0.05$ : a significant interaction effect

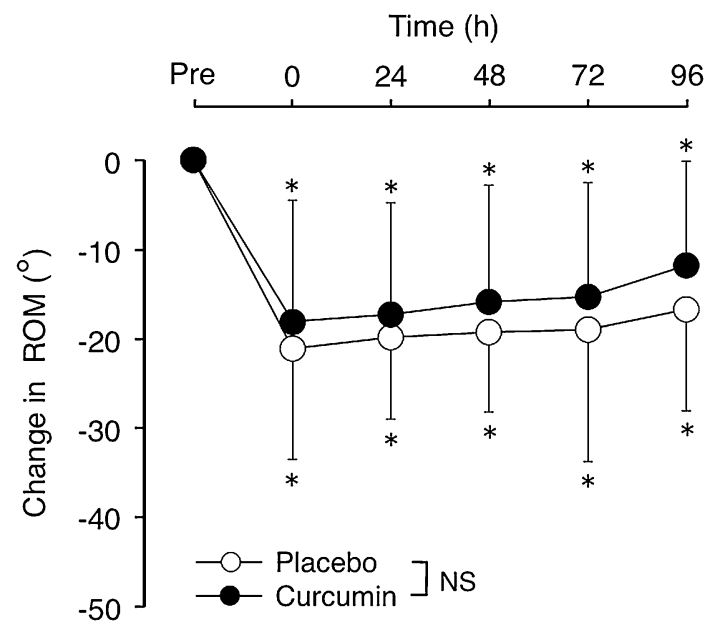

Fig. 3 Absolute changes (mean $\pm \mathrm{SD}$ ) in range of motion (ROM) from the baseline (Pre) at immediately (0) and 24-96 h after eccentric exercise of the elbow flexors for the curcumin and placebo supplementation conditions. $* P<0.05$ vs. Pre; $N S$ no significant interaction effect

\section{Plasma IL-6 and TNF- $\alpha$ concentration}

Plasma IL-6 and TNF- $\alpha$ concentrations were not different between groups before exercise (IL-6 $0.83 \pm 0.22$ vs. $0.73 \pm 0.18 \mathrm{ng} / \mathrm{mL}$, TNF- $\alpha 1.85 \pm 0.74$ vs. $1.63 \pm 0.35 \mathrm{ng} /$ $\mathrm{mL}$, for curcumin and placebo, respectively). They did not change significantly after eccentric exercise, and no significant differences were found between curcumin and placebo conditions (Fig. 7). 


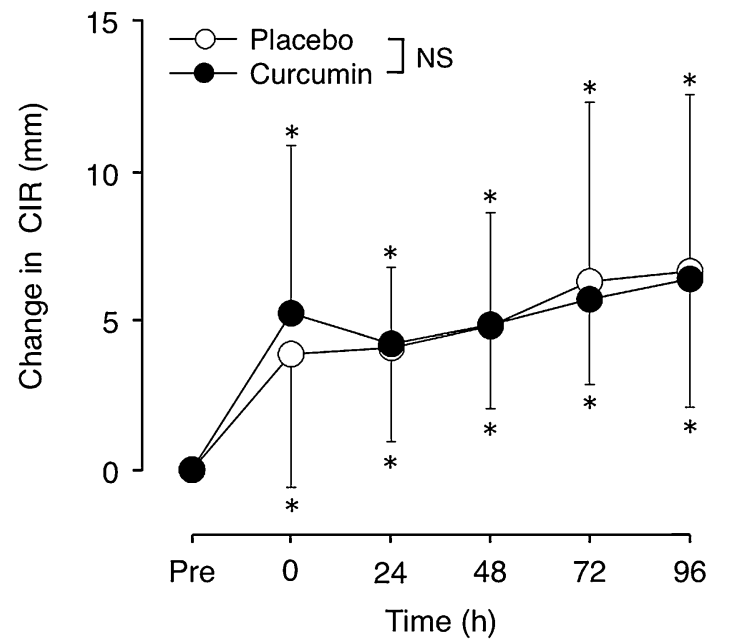

Fig. 4 Absolute changes (mean \pm SD) in upper-arm circumference (CIR) from the baseline (Pre) at immediately (0) and 24-96 h after eccentric exercise of the elbow flexors for the curcumin and placebo supplementation conditions. $* P<0.05$ vs. Pre; $N S$ no significant interaction effect

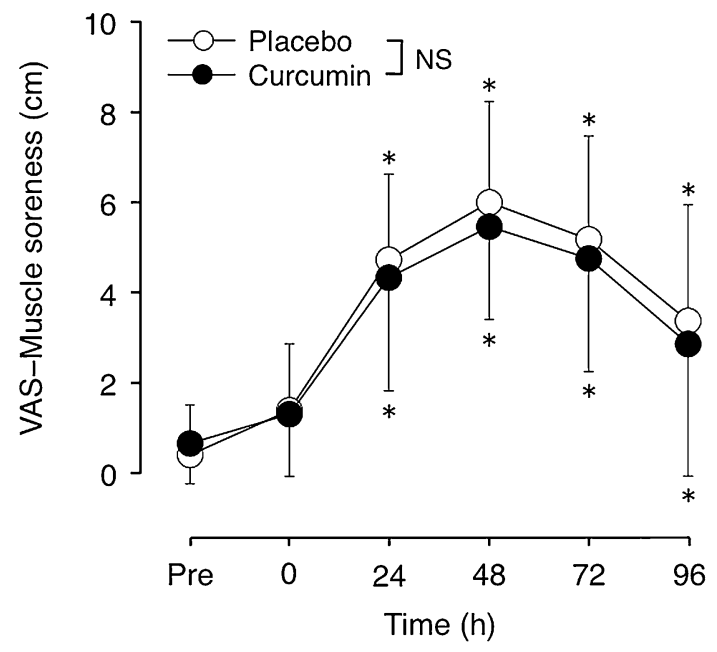

Fig. 5 Muscle soreness upon palpation of biceps brachii $($ mean $\pm \mathrm{SD}$ ) assessed by a visual analog scale $(10 \mathrm{~cm})$ before (Pre), immediately after (0) and 24-96 h after eccentric exercise of the elbow flexors for the curcumin and placebo supplementation conditions. $* P<0.05$ vs. Pre; $N S$ no significant interaction effect

\section{Discussion}

This study investigated whether curcumin (theracurmin) ingestion at $1 \mathrm{~h}$ before and $12 \mathrm{~h}$ after eccentric exercise would attenuate muscle damage in healthy young men. The main findings of the study include that (1) plasma curcumin concentration elevated from the baseline after exercise only for curcumin condition (Fig. 1); (2) the magnitude

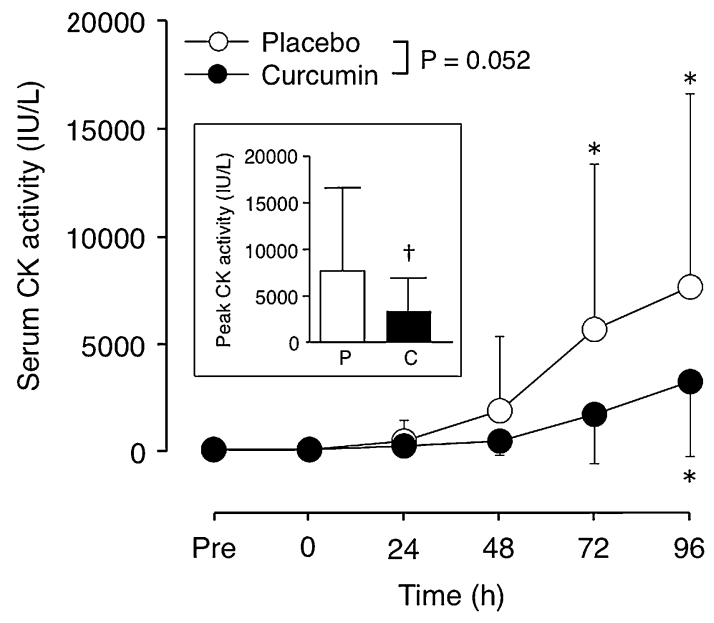

Fig. 6 Changes (mean \pm SD) in serum CK activity before (Pre), immediately after (0) and 24-96 h after eccentric exercise of the elbow flexors for the curcumin and placebo supplementation conditions. $* P<0.05$ vs. Pre; An inset, peak serum CK activity for the curcumin $(\mathrm{C})$ and placebo $(\mathrm{P})$ conditions is shown; ${ }^{\dagger} P<0.05$ vs. placebo
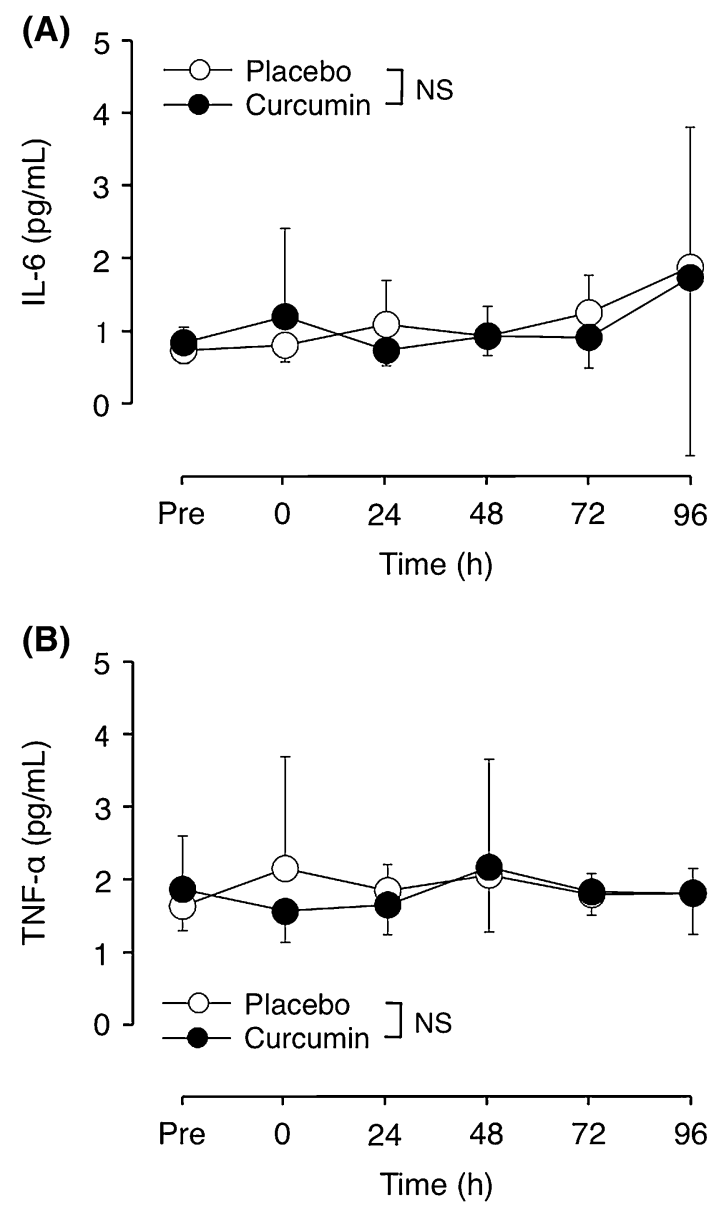

Fig. 7 Changes (mean \pm SD) in plasma IL-6 (a) and TNF- $\alpha$ (b) concentrations before (Pre), immediately after (0) and 24-96 h after eccentric exercise of the elbow flexors for the curcumin and placebo supplementation conditions. NS no significant interaction effect 
of decrease in MVC torque was significantly smaller for curcumin than placebo condition (Fig. 2), (3) peak serum CK activity was significantly reduced in the curcumin than placebo condition (Fig. 6), but (4) no significant differences between the conditions were evident for other variables (Figs. 3, 4, 5, 7). These results did not fully support the hypothesis that curcumin ingestion would attenuate the changes in the muscle damage markers after eccentric exercise, but showed that the curcumin was effective for some aspects of muscle damage.

The present study used an arm-to-arm comparison model, and the test order of curcumin and placebo conditions, as well as the use of the dominant and nondominant arms, were counterbalanced among subjects. The total work and mean peak torques during eccentric exercise did not differ between conditions. Although a small (7\%) non-significant difference in the total work was observed between the conditions, the total work did not correlate with the magnitude of decrease in MVC torque. Thus, it seems reasonable to expect that changes in the dependent variables were similar between the conditions, if curcumin ingestion had not produced any effects. However, the magnitude of decline in MVC torque immediately after exercise for the curcumin condition $(33 \%)$ was significantly smaller than that of the placebo condition ( $40 \%$ ), and this difference was sustained in the recovery days (Fig. 2). These indicate that the curcumin ingestion immediately before and/or $12 \mathrm{~h}$ after exercise affected the changes in MVC torque.

Trombold et al. (2010) showed that daily ellagitannin intake reduced loss of isometric elbow flexion strength by $10 \%$ at 48 and $72 \mathrm{~h}$ after exercise. Connolly et al. (2006) reported that cherry juice ingestion for 4 days before and for 4 days after exercise attenuated muscle strength loss by $20 \%$. These authors speculated that anti-inflammatory and/ or anti-oxidant effect of the substances decreased secondary muscle damage responses such as increases in vascular permeability, neutrophil and free radical, attenuating muscle fibre disruption (Connolly et al. 2006; Trombold et al. 2010). It is important to note that increases in serum CK activity were also attenuated by the curcumin ingestion in the present study (Fig. 6). Increases in CK activity in the blood indicate disruption of plasma membrane of muscle fibres and are commonly used as a marker of muscle fibre damage (Clarkson et al. 1992). It was reported that morphological disruption of myofibrils was associated with the magnitude of decrease in muscle function (Raastad et al. 2010). In the present study, the smaller increases in serum CK activity in the curcumin condition compared with the placebo condition may suggest that myofibril damage was attenuated by curcumin ingestion. This might be associated with the smaller decreases in MVC torque for the curcumin condition. Moriyuki et al. (2010) reported that curcumin inhibited production of prostaglandin via suppression of cyclooxygenase-2 upregulation and NF-kB signals, implying that curcumin ingestion might decrease vascular permeability. It may be that curcumin stabilises plasma membrane, which might be associated with the attenuated increase in serum CK activity and decrease in MVC torque after eccentric exercise. It is necessary to investigate the effects of curcumin on plasma membrane and myofibrils damage in future studies.

The present study found that curcumin ingestion had no positive effects on ROM (Fig. 3), swelling (Fig. 4), DOMS (Fig. 5) and blood markers (IL-6 and TNF- $\alpha$ ) of inflammation (Fig. 7). Changes in ROM, upper-arm circumference and DOMS after eccentric exercise were similar to those reported in previous studies (Evans et al. 2002; Nosaka et al. 2006). It was expected that curcumin ingestion would reduce the magnitude of decrease in ROM and increase in upper-arm circumference and DOMS; however, no such effects were found (Figs. 3, 4, 5). Davis et al. (2007) showed curcumin ingestion $(10 \mathrm{mg}$ ) in mice (considering the body weight difference, it would be $10,000 \mathrm{mg}$ in human) attenuated IL- 6 and TNF- $\alpha$ elevation following eccentric exercise. Comparing to this animal study, the amount of curcumin $(150 \mathrm{mg} \times 2)$ was small in the present study. However, it is known that curcumin is highly lipophilic and poorly bioavailabe, and that more than $8000 \mathrm{mg}$ of curcumin intake did not necessarily increase blood curcumin concentration in human (Kanai et al. 2012). In contrast, the theracurmin that was used in the present study was highly absorbable and more bioavailable when compared with conventional curcumin powder (Sasaki et al. 2011). It is not known how equivalent the amount of theracurmin used in the present study $(150 \mathrm{mg} \times 2)$ was to that of the previous studies that used conventional curcumin powder (e.g. $8000 \mathrm{mg}$ ). The subjects ingested theracurmin before exercise and $12 \mathrm{~h}$ after exercise, which resulted in prolonged increases in plasma curcumin concentration above the baseline for $96 \mathrm{~h}$ after exercise for the curcumin condition, although the level of plasma curcumin concentration was low $(8<\mathrm{ng} / \mathrm{mL})$ at $48-96 \mathrm{~h}$ post-exercise (Fig. 1). Although the range of the subject's body mass was relatively large $(50-91 \mathrm{~kg})$, there was no relationship between plasma curcumin concentration and body mass. A previous study showed that ingestion of theracur$\min (150 \mathrm{mg})$ increased plasma curcumin concentration already at $1 \mathrm{~h}$ post-ingestion, and its half-life time was $12 \mathrm{~h}$ in healthy adults (Kanai et al. 2012). Thus, it is assumed that the amount of curcumin for each time point $(150 \mathrm{mg})$ was adequate to provide anti-inflammatory effects, if the supplement had such effects. However, the present study found a significant effect of theracurmin supplementation before and after exercise on MVC torque and CK activity only, and no effects on ROM, muscle soreness and swelling. Nosaka et al. (2006) reported that essential amino acid 
supplementation given 30 min before and immediately after eccentric exercise (total of $7.2 \mathrm{~g}$ ) did not affect any indicators of muscle damage, but when the supplementation was continued in next 4 days after exercise (morning and evening for ten times over 5 days, total of $36 \mathrm{~g}$ ) attenuated increase in muscle soreness and ROM. Thus, it is possible that the effects of curcumin supplementation on DOMS and swelling would have been found, if the curcumin had been given on recovery days (e.g. 1-4 days post-exercise). There are many possible supplementation conditions in terms of dose, frequency and time points; a series of studies are warranted to investigate whether a larger dose and more frequent theracurmin supplementation in recovery days after eccentric exercise will provide greater effects on muscle damage.

The polyphenolic component of curcumin exerts antiinflammatory effects via downregulation of NF- $\mathrm{KB}$, which decreases in the expression of inflammatory cytokines such as IL-6 and TNF- $\alpha$ (Jobin et al. 1999; Shishodia et al. 2007; Davis et al. 2007). Thus, we expected to see smaller increases in IL- 6 and TNF- $\alpha$ after eccentric exercise for the curcumin than placebo condition; however, no significant differences between conditions were found, and they did not change significantly for either condition (Fig. 7). Hirose et al. (2004) showed no significant changes in IL-6 and TNF- $\alpha$ at 24-96 h after elbow flexor eccentric exercise and explained that the short exercise time and the small muscle mass involved in the exercise did not affect the inflammatory cytokine levels in the blood. Philippou et al. (2009) reported that knee extensor eccentric contractions increased serum IL- 6 concentrations at 6 and $48 \mathrm{~h}$ post-exercise, but Miles et al. (2008) showed that IL-6 increased at 8 and $12 \mathrm{~h}$, and returned to the baseline level at $24 \mathrm{~h}$ after elbow flexor eccentric exercise. Depner et al. (2010) reported that TNF- $\alpha$ concentration did not change, but IL- 6 decreased by $20 \%$ at 1.5 and $4 \mathrm{~h}$ after eccentric exercise of the elbow flexors and extensors. Thus, it might be that the present study missed the changes in IL- 6 and TNF- $\alpha$, because no blood samples were taken between immediately and $24 \mathrm{~h}$ after exercise. It is also interesting to assess other cytokines (e.g. IL-8, IL-10) and inflammatory markers (e.g. C-reactive protein) in future studies. Previous animal studies have reported that curcumin ingestion attenuated increases in inflammatory cytokine (e.g., IL-6 and TNF- $\alpha$ ) in the soleus muscle and oxidative stress markers (e.g., hydrogen peroxide and NADPH-oxidase) in the gastrocnemius muscle at 24 and $48 \mathrm{~h}$ after downhill running (Davis et al. 2007; Kawanishi et al. 2013). Therefore, it is also important to examine the effects of curcumin ingestion on inflammatory cytokines and oxidative stress marker in human skeletal muscles.

Many studies that investigated the effect of nutritional supplementation on muscle function have failed to find positive effect on muscle function (Nosaka et al. 2006;
Bryer and Goldfarb 2006; O'Fallon et al. 2012). However, the present study demonstrated that highly bioavailable curcumin attenuated the loss of muscle function after eccentric exercise. This may be important for athletes who train and compete regularly, since curcumin ingestion could help them to reduce prolonged loss of muscle function in an intense competition season. However, it should be noted that the present study used untrained men as the subjects and thus the findings of the present study cannot be generalised to other population such as women and trained individuals. Further studies are necessary to test women and trained individuals for the effects of curcumin supplementation on eccentric exercise-induced muscle damage. Furthermore, if curcumin supplementation provides strong anti-inflammatory effects, it should be investigated whether chronic curcumin intake attenuates muscle adaptations induced by resistance training. Trappe et al. (2001) reported that ibuprofen blunted protein synthesis after knee extensor eccentric exercise. Thus, it might be that chronic consumption of curcumin blunts muscle hypertrophy. The chronic supplementation of curcumin warrants further studies.

In conclusion, the present study demonstrated that the decrease in muscle strength and increase in serum CK activity after eccentric exercise were attenuated by highly bioavailable curcumin intake at $1 \mathrm{~h}$ before and $12 \mathrm{~h}$ after eccentric exercise of the elbow flexors in untrained men. These findings suggest that curcumin intake has some beneficial effects on recovery of eccentric exercise-induced muscle damage.

Conflict of interest A. I. was a consultant of the Theravalues Corporation, and Y. O. was an employee of the Theravalues Corporation, when the present study was conducted. This study was supported by the company.

Open Access This article is distributed under the terms of the Creative Commons Attribution 4.0 International License (http://creativecommons.org/licenses/by/4.0/), which permits unrestricted use, distribution, and reproduction in any medium, provided you give appropriate credit to the original author(s) and the source, provide a link to the Creative Commons license, and indicate if changes were made.

\section{References}

Aggarwal S, Ichikawa H, Takada Y, Sandur SK, Shishodia S, Aggarwal BB (2006) Curcumin (diferuloylmethane) down-regulates expression of cell proliferation and antiapoptotic and metastatic gene products through suppression of IkB kinase and Akt activation. Mol Pharmacol 69(1):195-206

Bryer SC, Goldfarb AH (2006) Effect of high dose vitamin C supplementation on muscle soreness, damage, function, and oxidative stress to eccentric exercise. Int J Sport Nutr Exerc Metab 16(3):270-280

Chen TC, Nosaka K (2006) Responses of elbow flexors to two strenuous eccentric exercise bouts separated by three days. J Strength Cond Res 20(1):108-116 
Cho JW, Lee KS, Kim CW (2007) Curcumin attenuates the expression of IL-1beta, IL-6, and TNF-alpha as well as cyclin E in TNF-alpha-treated HaCaT cells; NF-kappaB and MAPKs as potential upstream targets. Int J Mol Med 19(3):469-474

Clarkson PM, Nosaka K, Braun B (1992) Muscle function after exercise-induced muscle damage and rapid adaptation. Med Sci Sports Exerc 24(5):512-520

Connolly DA, Sayers SP, McHugh MP (2003) Treatment and prevention of delayed onset muscle soreness. J Strength Cond Res 17(1):197-208

Connolly DA, McHugh MP, Padilla-Zakour OI, Carlson L, Sayers SP (2006) Efficacy of a tart cherry juice blend in preventing the symptoms of muscle damage. Br J Sports Med 40(8):679-683

Davis JM, Murphy EA, Carmichael MD, Zielinski MR, Groschwitz CM, Brown AS, Gangemi JD, Ghaffar A, Mayer EP (2007) Curcumin effects on inflammation and performance recovery following eccentric exercise-induced muscle damage. Am J Physiol Regul Integr Comp Physiol 292(6):R2168-R2173

Depner CM, Kirwan RD, Frederickson SJ, Miles MP (2010) Enhanced inflammation with high carbohydrate intake during recovery from eccentric exercise. Eur J Appl Physiol 109(6): 1067-1076

Evans RK, Knight KL, Draper DO, Parcell AC (2002) Effects of warm-up before eccentric exercise on indirect markers of muscle damage. Med Sci Sports Exerc 34(12):1892-1899

Hirose L, Nosaka K, Newton M, Laveder A, Kano M, Peake J, Suzuki $\mathrm{K}$ (2004) Changes in inflammatory mediators following eccentric exercise of the elbow flexors. Exerc Immunol Rev 10:75-90

Howatson G, McHugh MP, Hill JA, Brouner J, Jewell AP, van Someren KA, Shave RE, Howatson SA (2010) Influence of tart cherry juice on indices of recovery following marathon running. Scand J Med Sci Sports 20(6):843-852

Jobin C, Bradham CA, Russo MP, Juma B, Narula AS, Brenner DA, Sartor RB (1999) Curcumin blocks cytokine-mediated NFkappa $\mathrm{B}$ activation and proinflammatory gene expression by inhibiting inhibitory factor I-kappa B kinase activity. J Immunol 163(6):3474-3483

Kanai M, Imaizumi A, Otsuka Y, Sasaki H, Hashiguchi M, Tsujiko K, Matsumoto S, Ishiguro H, Chiba T (2012) Dose-escalation and pharmacokinetic study of nanoparticle curcumin, a potential anticancer agent with improved bioavailability in healthy human volunteers. Cancer Chemother Pharmacol 69(1):65-70

Kawanishi N, Kato K, Takahashi M, Mizokami T, Otsuka Y, Imaizumi A, Shiva D, Yano H, Suzuki K (2013) Curcumin attenuates oxidative stress following downhill running-induced muscle damage. Biochem Biophys Res Commun 441(3):573-578

Miles MP, Andring JM, Pearson SD, Gordon LK, Kasper C, Depner CM, Kidd JR (2008) Diurnal variation, response to eccentric exercise, and association of inflammatory mediators with muscle damage variables. J Appl Physiol 104(2):451-458

Moriyuki K, Sekiguchi F, Matsubara K, Nishikawa H, Kawabata A (2010) Curcumin Inhibits the proteinase-activated receptor-2-triggered prostaglandin E2 production by suppressing cyclooxygenase- 2 upregulation and Akt-dependent activation of nuclear factor- $\kappa \mathrm{B}$ in human lung epithelial cells. J Pharmacol Sci 114(2):225-229
Newton MJ, Sacco P, Chapman D, Nosaka K (2013) Do dominant and non-dominant arms respond similarly to maximal eccentric exercise of the elbow flexors? J Sci Med Sport 16(2):166-171

Nosaka K, Newton M, Sacco P (2002a) Delayed-onset muscle soreness does not reflect the magnitude of eccentric exercise-induced muscle damage. Scand J Med Sci Sports 12(6):337-346

Nosaka K, Newton M, Sacco P (2002b) Muscle damage and soreness after endurance exercise of the elbow flexors. Med Sci Sports Exerc 34(6):920-927

Nosaka K, Sacco P, Mawatari K (2006) Effects of amino acid supplementation on muscle soreness and damage. Int J Sport Nutr Exerc Metab 16(6):620-635

O'Fallon KS, Kaushik D, Michniak-Kohn B, Dunne CP, Zambraski EJ, Clarkson PM (2012) Effects of quercetin supplementation on markers of muscle damage and inflammation after eccentric exercise. Int J Sport Nutr Exerc Metab 22(6):430-437

Paulsen G, Mikkelsen UR, Raastad T, Peake JM (2012) Leucocytes, cytokines and satellite cells: what role do they play in muscle damage and regeneration following eccentric exercise? Exerc Immunol Rev 18:42-97

Philippou A, Bogdanis G, Maridaki M, Halapas A, Sourla A, Koutsilieris M (2009) Systemic cytokine response following exercise-induced muscle damage in humans. Clin Chem Lab Med 47(6):777-782

Raastad T, Owe SG, Paulsen G, Enns D, Overgaard K, Crameri R, Kiil S, Belcastro A, Bergersen L, Hallén J (2010) Changes in calpain activity, muscle structure, and function after eccentric exercise. Med Sci Sports Exerc 42(1):86-95

Sasaki H, Sunagawa Y, Takahashi K, Imaizumi A, Fukuda H, Hashimoto T, Wada H, Katanasaka Y, Kakeya H, Fujita M, Hasegawa $\mathrm{K}$, Morimoto $\mathrm{T}$ (2011) Innovative preparation of curcumin for improved oral bioavailability. Biol Pharm Bull 34(5):660-665

Sethi G, Sung B, Kunnumakkara AB, Aggarwal BB (2009) Targeting TNF for treatment of cancer and autoimmunity. Adv Exp Med Biol 647:37-51

Shishodia S, Singh T, Chaturvedi MM (2007) Modulation of transcription factors by curcumin. Adv Exp Med Biol 595:127-148

Tiidus PM (1998) Radical species in inflammation and overtraining. Can J Physiol Pharmacol 76(5):533-538

Trappe TA, Fluckey JD, White F, Lambert CP, Evans WJ (2001) Skeletal muscle $\mathrm{PGF}_{2 \alpha}$ and $\mathrm{PGE}_{2}$ in response to eccentric resistance exercise: influence of ibuprofen acetaminophen. J Clin Endocrinol Metab 86(10):5067-5070

Trombold JR, Barnes JN, Critchley L, Coyle EF (2010) Ellagitannin consumption improves strength recovery 2-3 d after eccentric exercise. Med Sci Sports Exerc 42(3):493-498

Usharani P, Mateen AA, Naidu MU, Raju YS, Chandra N (2008) Effect of NCB-02, atorvastatin and placebo on endothelial function, oxidative stress and inflammatory markers in patients with type 2 diabetes mellitus: a randomized, parallel-group, placebocontrolled, 8-week study. Drugs R D 9(4):243-250

Warren GL, Lowe DA, Armstrong RB (1999) Measurement tools used in the study of eccentric contraction-induced injury. Sports Med 27(1):43-59 